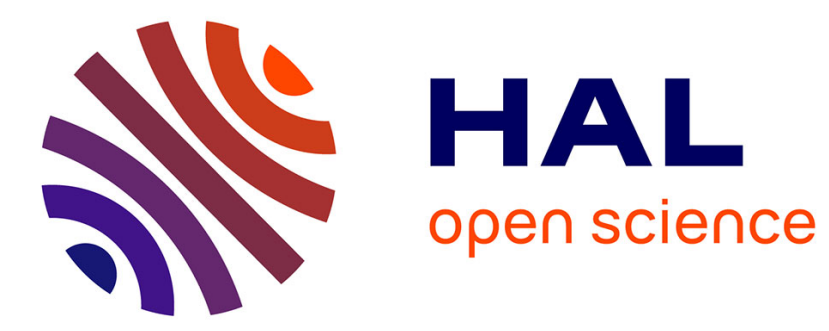

\title{
Building and Editing a Sealed Geological Model
}

Guillaume Caumon, François Lepage, Charles H Sword, Jean-Laurent Mallet

\section{To cite this version:}

Guillaume Caumon, François Lepage, Charles H Sword, Jean-Laurent Mallet. Building and Editing a Sealed Geological Model. Mathematical Geology, 2004, 36 (4), pp.405 - 424. 10.1023/b:matg.0000029297.18098.8a . hal-03029470

\section{HAL Id: hal-03029470 \\ https://hal.univ-lorraine.fr/hal-03029470}

Submitted on 28 Nov 2020

HAL is a multi-disciplinary open access archive for the deposit and dissemination of scientific research documents, whether they are published or not. The documents may come from teaching and research institutions in France or abroad, or from public or private research centers.
L'archive ouverte pluridisciplinaire HAL, est destinée au dépôt et à la diffusion de documents scientifiques de niveau recherche, publiés ou non, émanant des établissements d'enseignement et de recherche français ou étrangers, des laboratoires publics ou privés. 


\title{
Building and Editing a Sealed Geological Model ${ }^{1}$
}

\section{Guillaume Caumon ${ }^{2,3}$, François Lepage ${ }^{2}$, Charles H. Sword ${ }^{4}$ and Jean-Laurent Mallet ${ }^{2}$}

\begin{abstract}
In Solid Modeling, a boundary representation (b-rep) defines solids by their bounding surfaces, providing an efficient volume description. Building on this representation, we present the notion of a Sealed Geological Model. In such a model, the geological surfaces define a partition of the domain of interest into regions; analytic functions can be defined in these regions to describe the spatial variations of the subsurface properties. Such descriptions can be used in Geophysics, $3 D$ GIS, and for discretization purposes. In addition to the b-rep representational validity conditions, Sealed Geological Models must satisfy conditions of geological consistency. Bearing these conditions in mind, we describe a methodology to create and modify the shape of such sealed models interactively. We use the hierarchical relationship between geological surfaces to help reshape the contact between a fixed surface (a surface that other surfaces can slide along, such as a fault, erosion surface, or salt top) and a secondary deformable surface (e.g. horizon, older fault). Although designed to meet the demanding requirements of interactive model editing, our methodology could also make use of displacement vectors computed by an automatic process such as tomographic inversion or 3D balanced unfolding.
\end{abstract}

KEY WORDS: Geomodeling, Solid Modeling, Boundary Representation, Validity, Algorithms.

\section{INTRODUCTION}

Integrated modeling of the Earth's interior involves a variety of representations to describe the geometry of geological objects, their relative relationships and the properties of their rock units. Among these representations, solid representations (Mäntylä, 1988, Hoffmann, 1989) are of particular interest to geoscientists (Gjøystdal, Reinhardsen, and Åstebø1, 1985, Turner, 1992; Bonham-Carter, 1994;, Halbwachs and others, 1996, Mello and Henderson, 1997; Assa, Celnicker, and Ramshorn, 1998; Mallet, 2002), in that they allow us to consider the subsurface as a collection of distinct volumes, whose interior and exterior are unambiguously defined.

\footnotetext{
${ }^{1}$ Published as Caumon et al. (2004), Mathematical Geosciences 36(4):405-424 doi:10.1023/B:MATG.0000029297.18098.8a

${ }^{2}$ ENSG-INPL-CRPG, 54501 Vandoeuvre-Lès-Nancy, France; email: [Guillaume.Caumon; Francois.Lepage; JeanLaurent.Mallet]@ensg.inpl-nancy.fr

${ }^{3}$ Presently at: Department of Petroleum Engineering, Stanford University, Stanford, CA 94305-2220; email: gcaumon@pangea.stanford.edu

${ }^{4}$ ChevronTexaco EPTC, 4800 Fournace Place Bellaire, TX 77401; email: chsword@ chevrontexaco.com
} 
Raster data structures (Cartesian grids or octrees) have been widely used to represent geological units (Turner, 1992; Bonham-Carter, 1994), because they are efficiently represented on computers. Yet, the very nature of geological objects calls for more flexible representations, capable of handling, e.g., complex stratigraphies, intricate fault networks, and salt geometries. The concept of Boundary Representation (b-rep) provides the necessary versatility. In this approach, a volume object is entirely defined by its boundary, which can consist of any type of closed and orientable free-form surface.

During recent decades, several topological representations have been proposed to model geological structures with b-reps (Gjøystdal, Reinhardsen, and Åstebø1, 1985, Lamboglia, 1994, Halbwachs and others, 1996. Mello and Henderson, 1997, Lévy, 1999, Euler, Sword, and Dulac, 1999, Hubeli, 2002). Although most provide the required flexibility, actual models have proven difficult to build and update. This is due to a lack of specificity of what the fundamental features of a geological model should be.

In this article, we address this problem by introducing two geological validity criteria on top of the classical b-rep validity conditions. Because these criteria restrict the class of possible b-reps, we call this new representation a Sealed Geological Model. We then propose a means of building and editing such a model.

\section{FROM BOUNDARY REPRESENTATION (B-REP) TO SEALED GEOLOGICAL MODEL \\ Principle}

A geological map represents geological units by their boundaries. Extending this idea to 3D models is conceptually straightforward: geological interfaces (horizons, unconformities, faults, evaporite boundaries, etc.) create a partition of space into well-defined polyhedral regions.

Although this region-wise model description is quite powerful, it is tedious to use in geological applications. Therefore, several authors (Sword, 1991; Lamboglia, 1994; Mello and Henderson, 1997; Assa, Celnicker, and Ramshorn, 1998) propose to group correlated regions into layers (grouping together regions having the same stratigraphic age or lithology) and fault blocks (identifying the foot-wall and hanging-wall of a fault). Rock properties are defined by analytical functions within each region or set of regions.

From this basic principle, several topological representations have been proposed.

\section{Topological Model}

In their pioneering work, Gjøystdal, Reinhardsen, and Åstebøl (1985) split the space by a set of orientable parametric surfaces representing geological interfaces. The model consists of a tree of boolean operations on the resulting half-spaces. This method is very flexible, but requires a finite resolution grid to evaluate the 


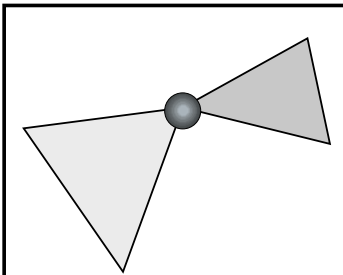

A

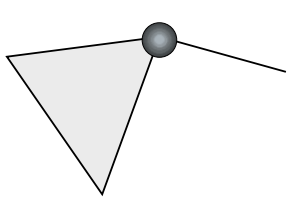

B

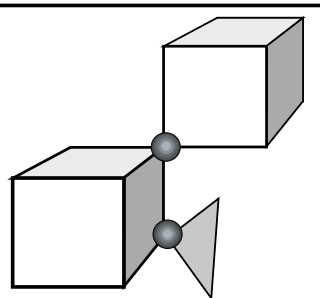

$\mathrm{C}$

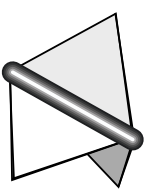

$\mathrm{D}$

Figure 1. Some examples of non-manifold surface configurations. The non-manifold singularities are highlighted. Non-manifold edges (D) are needed in geological models to represent contacts between geological interfaces.

regions. Moreover, maintaining the model topology over time is difficult because the topological representation of the regions is not explicit. It is now well known (Weiler, 1985; Mäntylä, 1988; Bonham-Carter, 1994; Mallet, 2002) that the geometry of a model must be accompanied by a complete topological description of this model.

Most topological representations are based on the concept of cellular complexes (Lévy, 1999, Mallet, 2002, de Floriani, Morando, and Puppo, 2003). A $n$-dimensional cellular complex $\Omega$ on a set $C_{0}$ of vertices is a set of $k$-cells, $0 \leq k \leq n$. A $k$-cell is a subset of $C_{0}$, homeomorphic to an open $k$-ball. The boundary $\partial c$ of a $k$-cell $c$ is defined as the set of $l$-cells, $0 \leq l<k$ that are proper subsets of $c$. The $(k-1)$-cells in the boundary of a cell $c$ are called faces of $c$. In addition, $\Omega$ is such that:

$$
\forall v \in C_{0}, \quad v \in \Omega
$$

And, denoting the boundary of $c$ by $\partial c$ :

$$
\forall c \in \Omega, \quad \partial c \subset \Omega
$$

A 2D cellular complex for which the neighborhood of each point is homeomorphic to an open disc is said to be manifold. Any surface that does not honor this property is non-manifold (Fig. 1). In geological models, surfaces with non-manifold edges (Fig. 11-D) are of particuliar interest, e.g., to represent the junction between two faults or between a horizon and a fault.

For that, a first category of approaches (Lamboglia, 1994, Mello and Henderson, 1997) use the notion of radial edge (Weiler, 1985) to represent these non-manifold singularities.

Halbwachs and others (1996) propose to model a fault network directly with a 3D data structure: a Generalized Map (3-G-Map) (Lienhardt, 1989). The incremental construction method first computes the intersections of a fault $F$ with the other surfaces of the model. The relationships between $F$ and these surfaces are then used to create fault polygons, that are inserted in the 3-G-Map by means of well-defined topological operators. This method provides a comprehensive topological definition of the fault blocks, but only handles planar fault geometries. 
In contrast, the hierarchical approaches first introduced by de Floriani and Falcidieno (1988) can efficiently represent complex fault or horizon geometries. These methods are based on a distinction between the micro-topology, which characterizes each surface separately using a 2-manifold data structure, and the macrotopology, which describes how these surfaces are associated along their boundaries to make up volume regions. Both radial-edge based data structures (Sword, 1991; Lamboglia, 1994; Euler, Sword, and Dulac, 1998) and 3D data structures (Hubeli, 2002; Lévy, 1999, Mallet, 2002) use this type of hierarchy.

Although these approaches provide the required flexibility, the quest for a perfect representation is not yet over (de Floriani, Morando, and Puppo, 2003). In particular, even the otherwise complete algebraic specifications of G-Maps (Lienhardt, 1989, Halbwachs and others, 1996) and of Hierarchical G-Maps (Lévy, 1999. Mallet, 2002) cannot properly tackle polyhedral regions embedded within other regions. For that, an auxiliary inclusion tree has to be added to the data structures.

To prove the effectiveness of the concepts developed in this work, we have used the hierarchical representation similar to that of Lamboglia (1994). A model $\mathcal{M}=\{\mathcal{S}, \mathcal{E}, \mathcal{R}, \mathcal{L}, \mathcal{F}\}$ is defined by:

- A set of triangulated manifold surfaces $\mathcal{S}=\left\{B, F_{0}, \ldots, F_{n_{f}}, H_{0}, \ldots, H_{n_{h}}\right\}$, representing the geological interfaces. These interfaces consist of a domain boundary $B$, layer boundaries (horizons) $H_{i}$ and faults $F_{i}$. These surfaces are subdivided into triangles (Mallet, 1992, 2002), represented by a half-edge based data structure (Preparata and Shamos, 1985).

- A set of radial edges $\mathcal{E}$ (Weiler, 1988), which associate border triangles of $\mathcal{S}$ whose edges coincide.

- The list of polyhedral regions $\mathcal{R}$ defined by this association of surface patches. Each region $R \in \mathcal{R}$ is uniquely defined by its internal and external bounding interfaces, and can be assigned a name in a deterministic manner.

- Groupings of regions into layers $\mathcal{L}$ and fault blocks $\mathcal{F}$. For each group $G \in \mathcal{L} \cup \mathcal{F}$, an analytical function can be defined to describe the variations of a property within the layer or fault block.

This topological model has the required representative power to deal with geological models. In that sense, it is equivalent to the models described by Lévy (1999); Mallet (2002); Assa, Celnicker, and Ramshorn (1998); Hubeli (2002). The methods and the models presented in this article could thus be easily translated into one of these topological models.

In this work, we are mostly concerned by the creation and the editing of b-reps of geological structures. Therefore, we need first to consider the validity of the object, both from the representational and the geological standpoint. 


\section{Representational Validity}

In the Solid Modeling literature, a great deal of care has been taken to specify the representational validity of a b-rep (Mäntylä, 1988, Sakkalis and Shen, 2000, Higashi and others, 2001). A model is considered invalid if does not yield a physical object in 3D space (for instance, a Klein bottle does not define the boundary of a valid solid). In the ideal modeling software, the following conditions should be observed at all times:

- A solid model has a finite extent in space. In practice, this requires that a bounding box can be found for the model.

- Any two distinct $i$-cells may intersect only at common $(i-1)$-cells belonging to their boundaries (nonintersection condition). In practice, this requires that the topological data structures be consistent with the geometry of the model.

The main cause of violations of the non-intersection condition is failure of the surface intersection computation. Reliable algorithms for computing surface intersections are difficult to come up with because of the limited precision of floating point numbers in computers (Fortune, 1995). Therefore, as noted by Higashi and others (2001), defining the topology of a b-rep cannot rely only on geometrical computations, but on additional constraints. In CAD applications, these constraints are usually provided by a parametric definition which is not suited to all geological objects (Mallet, 1997, 2002)

\section{Sealed Geological Model}

Consider the set of manifold surfaces $\mathcal{S}$ of a model $\mathcal{M}=\{\mathcal{S}, \mathcal{E}, \mathcal{R}, \mathcal{L}, \mathcal{F}\}$. A subset $\beta(\partial S)$ of a surface boundary $\partial S, S \in \mathcal{S}$ is said to be a free border if it is not bound to another surface by any radial edge of $\mathcal{E}$.

From this definition, two fundamental conditions for $\mathcal{M}$ are needed for geological validity :

Condition 1 Only faults may have free borders, corresponding to a null displacement of blocks. The borders of the other types of surface should be bound to target surfaces :

$$
\forall S \in \mathcal{S}, \quad \exists \beta(\partial S) \subset \partial S \text { such that } \beta(\partial S) \text { is free } \quad \Rightarrow \quad S \text { is a fault }
$$

Condition 2 Any two distinct layer boundaries $\mathcal{H}_{i}$ and $\mathcal{H}_{j}$ cannot cross each other: by definition, one of the oriented faces of a layer boundary identifies one and only one layer; thus, if $\mathcal{H}_{i}$ and $\mathcal{H}_{j}$ could cross each other, a region of space would belong to two layers, which is impossible.

A b-rep that honors these two constraints is a sealed geological model (Fig. 2). Note that an invalid b-rep 


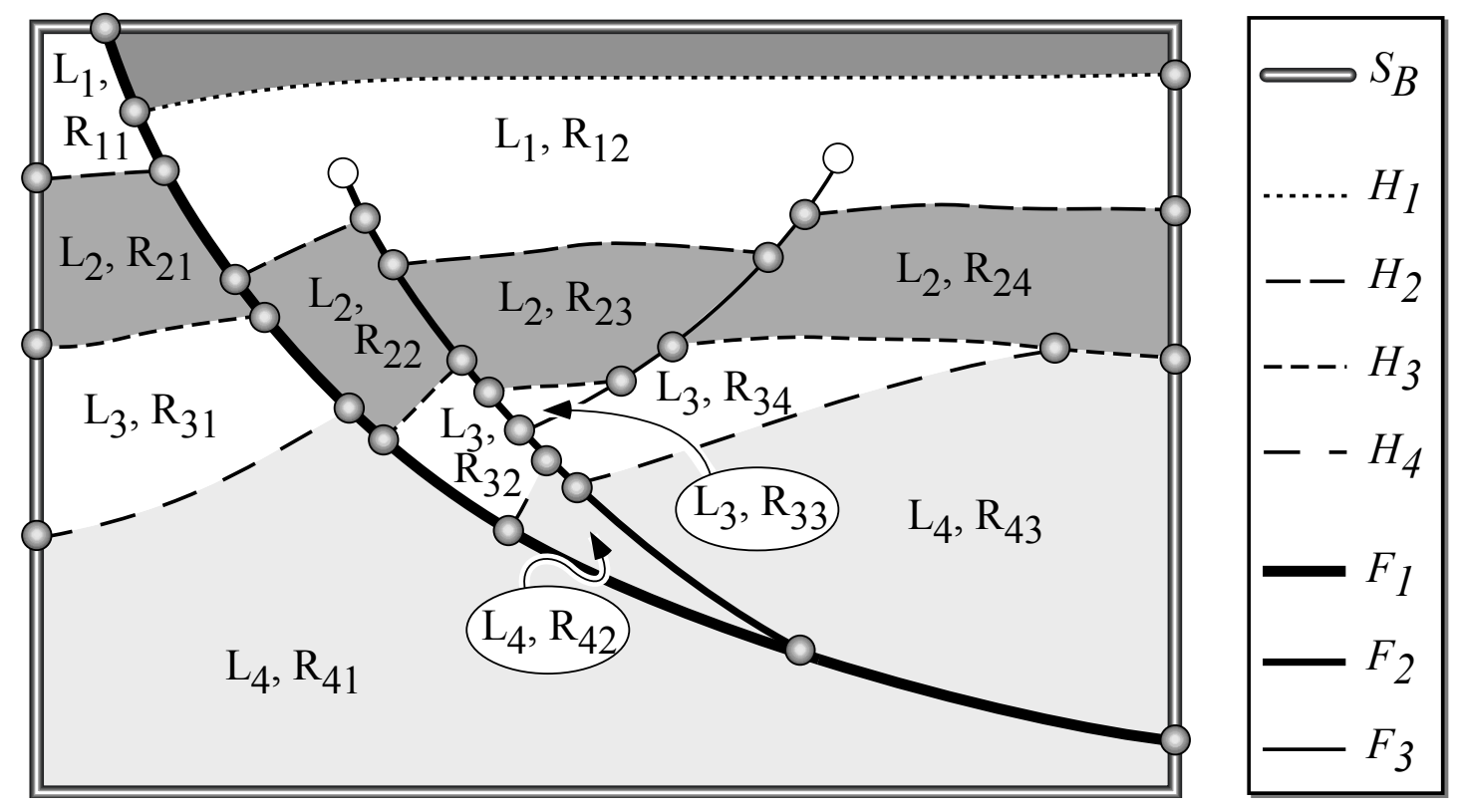

Figure 2. A cross-section view of a valid geological model $\mathcal{M}$, made of a domain boundary $S_{B}$, three faults $\left\{\mathcal{F}_{1}, \mathcal{F}_{2}, \mathcal{F}_{3}\right\}$ and four layer boundaries $\left\{\mathcal{H}_{1}, \mathcal{H}_{2}, \mathcal{H}_{3}, \mathcal{H}_{4}\right\}$. The model also contains the list of regions making up the layers. For instance, layer $L_{4}$ consists of the regions $R_{41}, R_{42}$ and $R_{43}$. Surface borders are denoted by circles.

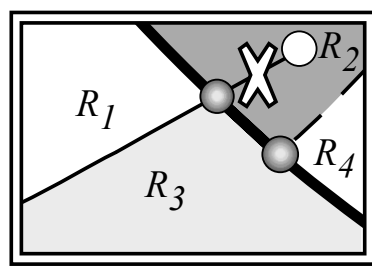

A

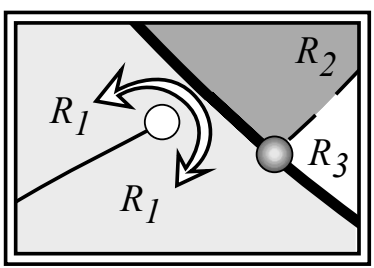

B

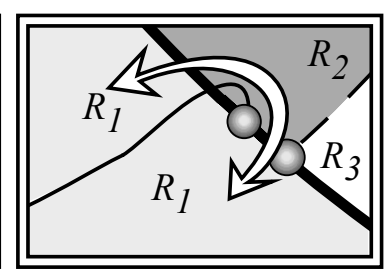

C

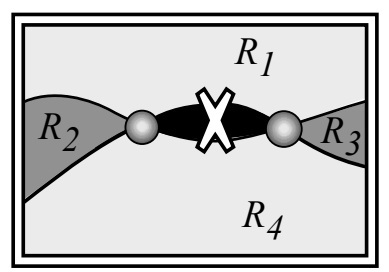

D

Figure 3. Detailed view of invalid features commonly generated by cut-based approaches. Horizons are denoted by light lines, and faults by bold lines; free surface borders are denoted by white circles. Free horizon borders are related to dangling horizon parts (A) or to leaks between layers (B). Such leaks may also be due to errors in the cut algorithm (C). Overlapping layers (D) violate the second validity condition.

(Fig. 3-C) is always an invalid sealed geological model; the converse is not true, since legal b-rep features may violate geological consistency (Fig. 3.A, B, D).

These minimal conditions can be checked using topological information, provided that the model $\mathcal{M}$ has a valid representation. These conditions are general, and mass conservation, deformation and rupture mechanisms (Ramsay and Lisle, 2000), or more advanced criteria (Gratier and Guillier, 1993, Thibault and others, 1996) could be formulated allowing a better characterization of b-rep validity. These minimal conditions can 


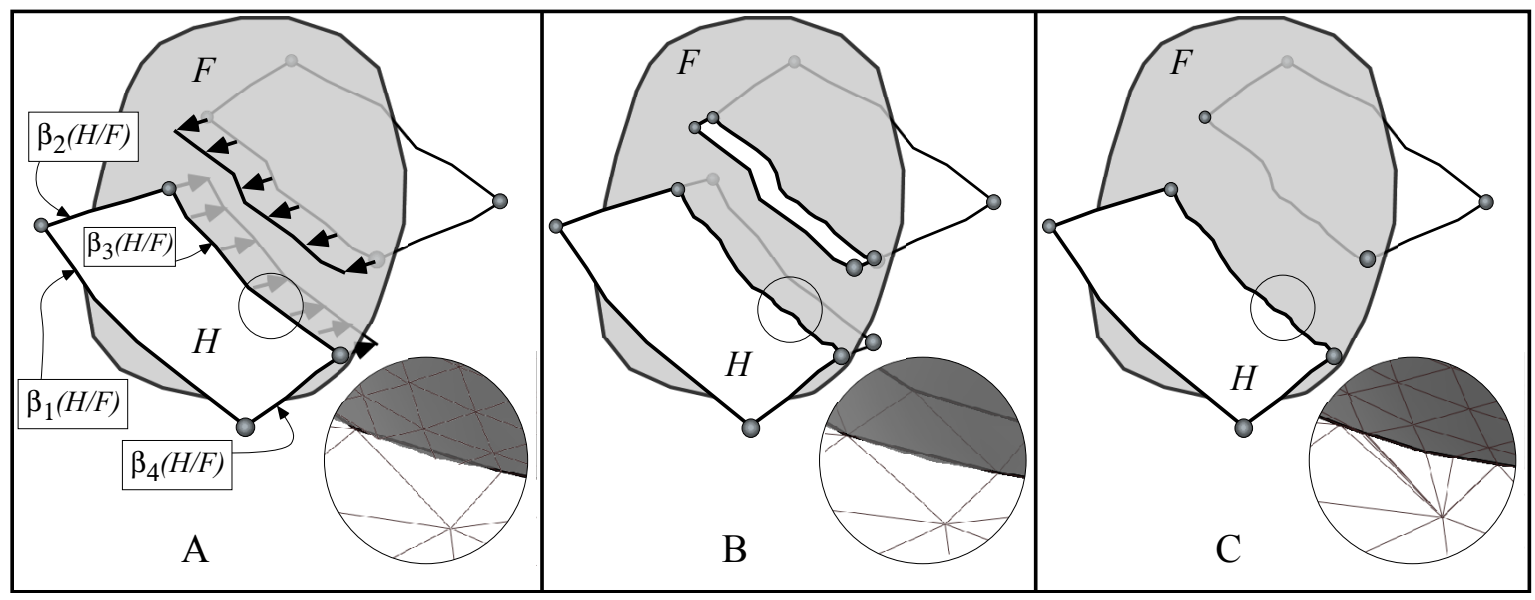

Figure 4. Cut-based approach for model building. From a structural model with borders consistently defined (A), the horizon borders are extrapolated (B). After the cut, the dangling horizon parts need to be removed (C). Note the poor mesh quality of the resulting surfaces.

be used to automatically detect invalid features, a preliminary step for any model correction.

Together with the representational validity constraints of a b-rep, these geological validity constraints define a Sealed Geological Model. The remainder of this article describes how to build and edit interactively such a model.

\section{BUILDING A SEALED MODEL}

We propose a process to build a Sealed Geological Model from a set of triangulated surfaces. The interfaces have been interpolated from available data as decribed by Mallet (1997), and are discontinuous across faults. The boundary $\partial S$ of a surface $S$ of $\mathcal{S}$ is subdivided into a set of contiguous borders. A border $\beta(\partial S)$ consists of a polygonal line whose vertices, called nodes, have the same status in the model. Using a "Boundary on surface" constraint (Mallet, 2002, Chap. 6), the nodes of a border $\beta\left(\partial S / S_{\text {target }}\right)$ can be constrained to lie on a target surface $S_{\text {target }}$, e.g., a fault (Fig. 4.A). Using this information, the goal is to generate a sealed geological model, i.e., a b-rep that honors the representational and geological constraints.

\section{Previous Work}

Classical methods to build boundary representations are based on surface cut algorithms (Fortune, 1995, Halbwachs and others, 1996). The geometrical intersections between the surfaces of $\mathcal{S}$ are computed, and used to bind surfaces together.

Unfortunately, this approach cannot be applied directly in most cases, and a prior extrapolation of surfaces is often needed to avoid leaks between regions (Fig. 4). For instance, horizon borders have to be extrapo- 
lated across faults, unconformities and domain boundaries. The free surface parts on the other side of the discontinuity must be removed after the cut operation. This tedious process could be automated by removing automatically free surface parts according to Constraint 1.

Instead of extrapolating surfaces, Euler, Sword, and Dulac (1998) propose an automatic approach to cut surfaces under constraints. The operation exploits the "boundary on-surface" constraint: the nodes of a border $\beta\left(\partial S / S_{\text {target }}\right)$ are projected onto the target surface $S_{\text {target }}$; the shortest path between two consecutive nodes is then used to compute the intersection line. This method is a significant improvement over previous approaches, but relies on intersection computations.

Due to the complexity of the algorithms involved, these cut-based approaches may not always yield a valid model. The most frequent symptoms of invalidity are leaks between two regions (infringement of condition 1, Fig. 3-B-C) or the existence of "bubbles", which have no geological meaning (infringement of condition 2 , Fig. 3.D).

Because the cut operation refines the mesh of the intersecting surfaces along the intersection curve, the resulting border triangles may have a poor aspect ratio (Fig. 4.C), which may cause numerical problems in subsequent modeling operations.

Recently, two elegant approaches based on Voronoi diagrams have been proposed. Courrioux and others (2001) creates volume models from field observations and geological surfaces: points are generated on both sides of each interface using information about the surface normals; the Voronoi diagram of these nodes is then smoothed and Voronoi polyhedra having the same geological nature are merged. Hale and Emanuel (2002) proposes a physical model to displace nodes within a seismic image; the resulting Voronoi diagram is aligned on the seismic features, and can be used as a reservoir simulation grid and to improve seismic interpretation.

\section{Construction Method}

The validity conditions introduced in the first Section make it possible to consider another approach to model building. Instead of deducing the model topology from geometrical computations, we propose to conform directly to the geological validity constraints.

Several ideas underlie this method:

- The boundary $\partial S$ of a surface $S \in \mathcal{S}$ is subdivided into borders $\left\{\beta_{1}(S), \ldots, \beta_{k}(S)\right\}$. If $S$ is not a fault, all borders must be embedded into target surfaces to meet the first geological validity condition. This can be done either automatically, using proximity queries, or interactively. Either way, it is simple to enforce that all surfaces except faults have no free borders. 

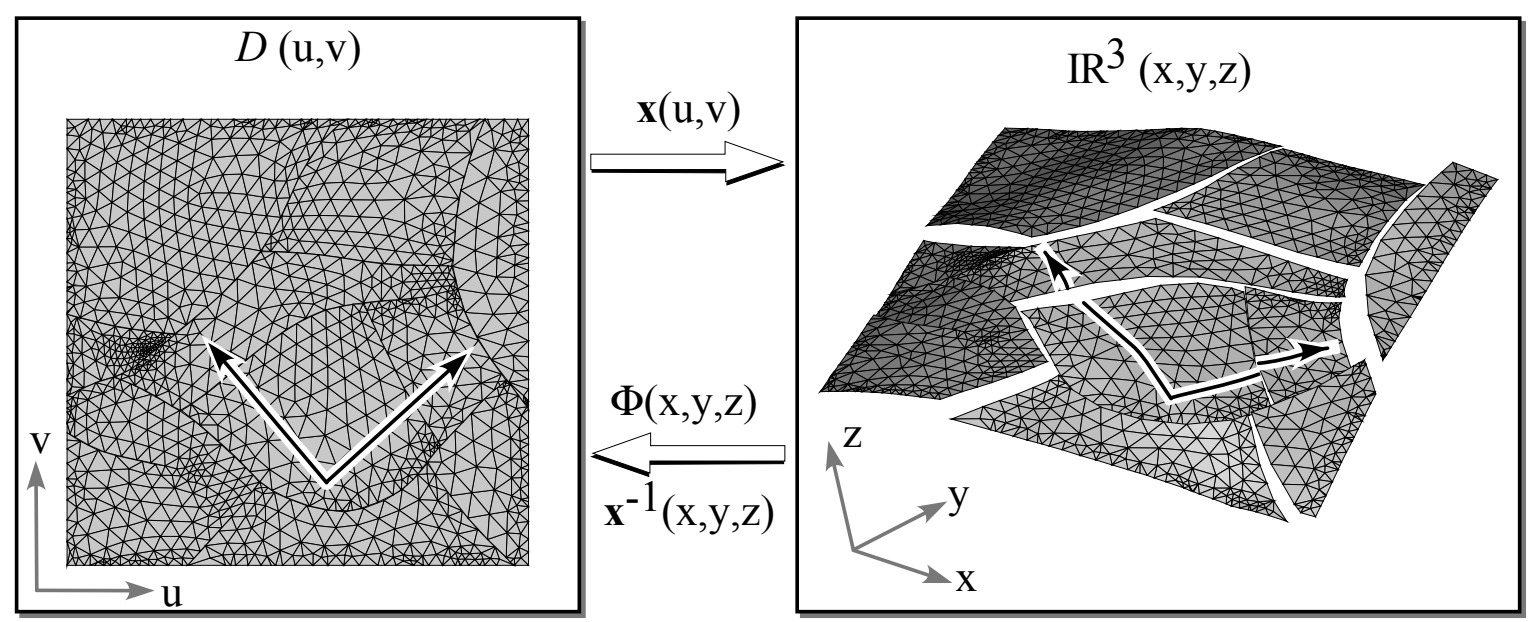

Figure 5. Parameterization $\mathbf{x}(u, v)$ and inverse parameterization $\phi(x, y, z)$ defined on a triangulated surface.
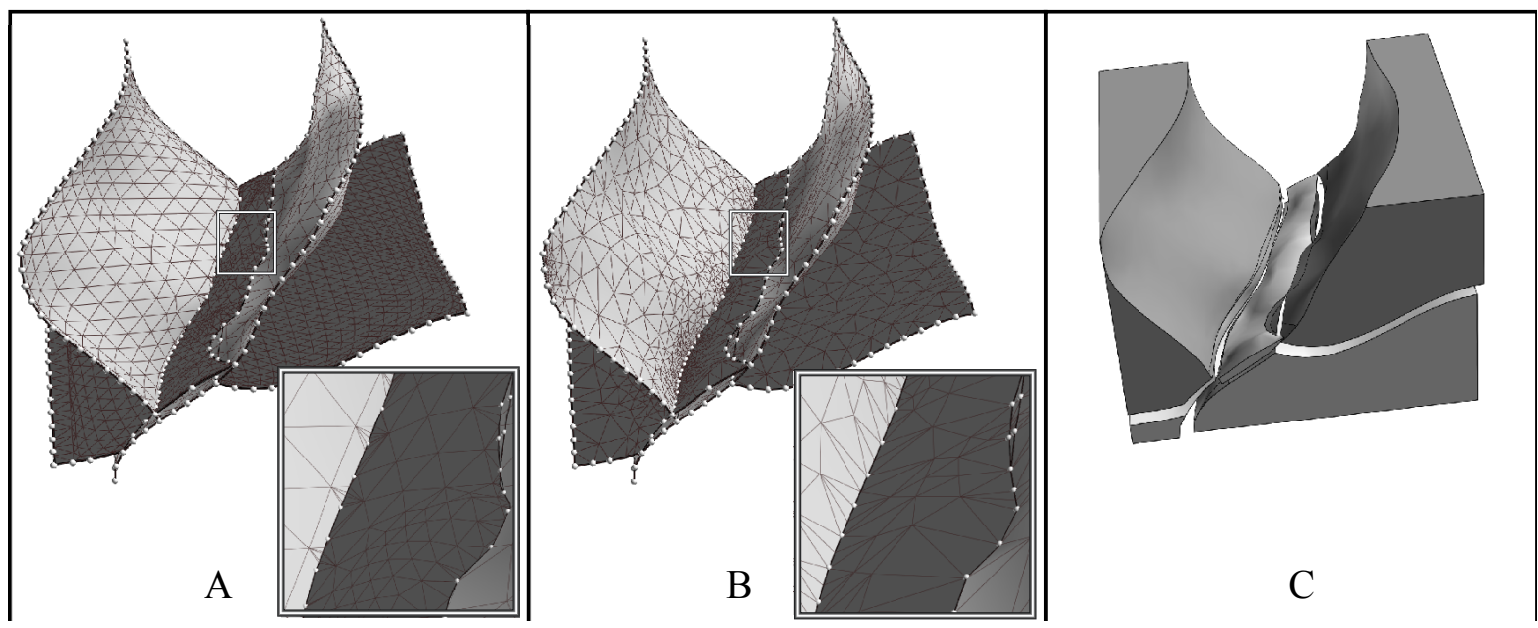

Figure 6. Proposed construction method. The borders of the initial surfaces are evenly resampled (A). The interfaces are then retriangulated to conform to these radial lines (B), and disconnected to set the radial edges (exploded view, $\mathrm{C}$ ).

- Any surface $S$ of $\mathcal{S}$ can be piecewise parameterized by a bijective function $\mathbf{x}(u, v)$ such that

$$
\forall(u, v) \in \mathcal{P} \Rightarrow \mathbf{x}(u, v) \in S
$$

where $\mathcal{P}$ is the parametric domain of $S$ in the plane $\mathbb{R}^{2}$ (Fig. 5). The function $\mathbf{x}(u, v)$ should be such as to minimize distortions, see Lévy and Mallet (1998); Lévy and others (2002).

From these considerations, the construction method proceeds as follows (Fig. 6): 
1. The contact lines between surfaces are computed from the constraints on borders, yielding a frame model (Lepage, 2002).

2. The frame model is resampled evenly and each surface of the model is resampled consistently along contacts using a conforming Delaunay triangulation (Lepage, 2002).

3. Surfaces are disconnected along the contact lines, and radial edges are created along each border edge. The Sealed Geological Model is then built by traversing the surface patches using these non-manifold radial edges.

As compared to the cut-based approaches, this method uses only the constraints on surface borders to create the model. Therefore it is assumed that any intersection between the initial surfaces is represented by a constrained border.

\section{MODEL EDITING}

A numerical model of the subsurface is never more than a mere approximation of reality. When new information becomes available, e. g., when a well is drilled, it is thus common to observe a misfit between the old model and the new observations. In most cases, editing an existing model to account for the new information is much preferable to a complete model rebuild. When the initial model is built from field data, editing is also needed after a first interpretation (de Kemp, 1999).

\section{Previous Work}

Editing parts of a solid model may imply topological changes. At present, most approaches described in the literature consider only deformations that do not modify the macro-topology of the model (Mello and Henderson, 1997; Hubeli, 2002). For instance free-form deformation (FFD), introduced by Sederberg and Parry (1986), uses a smooth spatial deformation function applied to the whole model.

With regard to the two validity conditions presented above, FFD methods can give good results, provided that the discretization of the deformation function is carefully handled. However, for many applications, it is desirable to leave room for controlled topological changes.

Gjøystdal, Reinhardsen, and Åstebø1 (1985) propose to modify oriented free-form surfaces and recompute the model definition using a grid and boolean operations. Euler, Sword, and Dulac (1999) describe how an interface can be removed from the model, globally modified under geometric constraints, and reincorporated into the volume model using a surface "cut with constraints" operation that honors contacts. This operation is general and flexible, but requires a complete reconstruction of the model. The process is computationally 


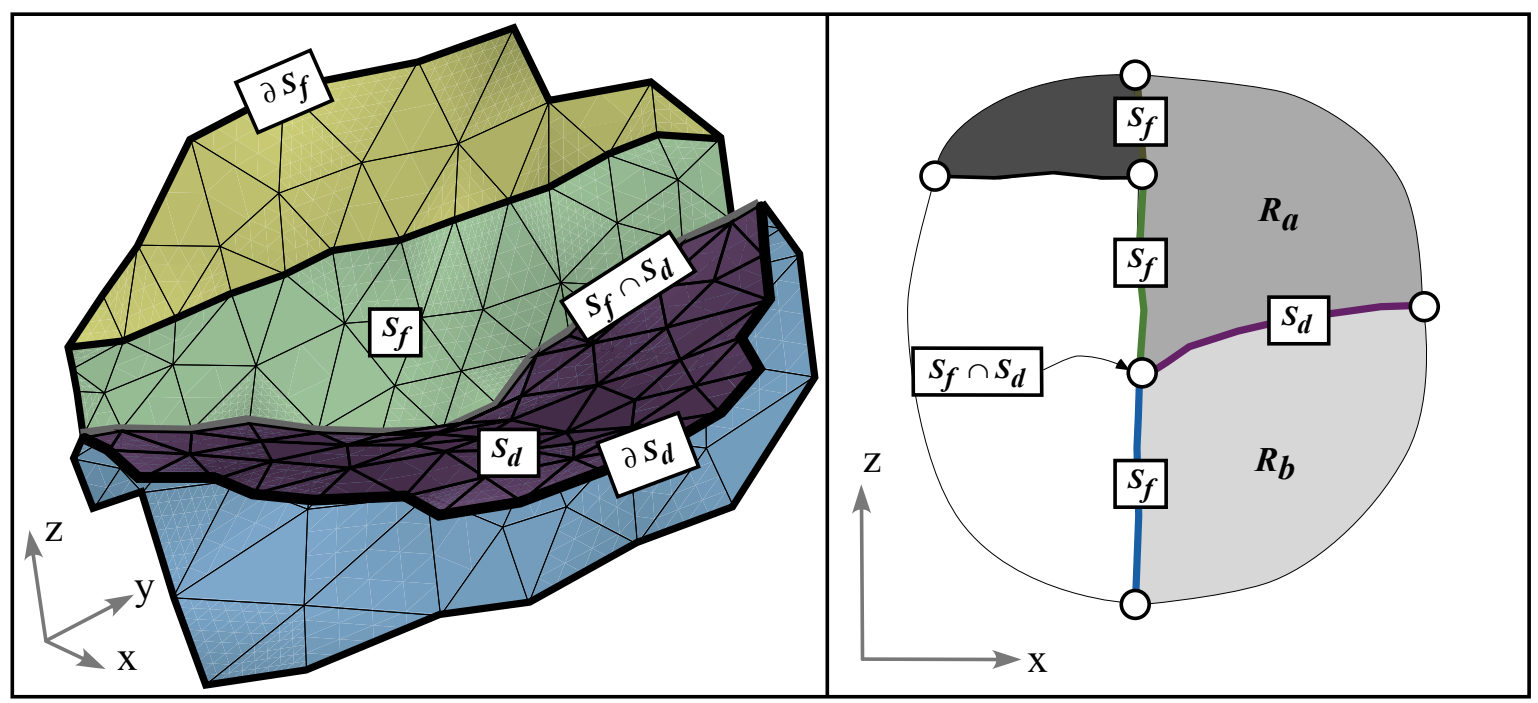

Figure 7. The sets defined in the modified domain. The simplices of the domain are classified according to their belonging to the interior or the border of the fixed surface $S_{f}$ or of the surface to deform $S_{d}$.

expensive, and does not guarantee the persistence of geological invariants (number of regions, definition of layers, etc.).

\section{Method outline}

Our editing method displaces in real time the interfaces of a sealed model $\mathcal{M}$ while maintaining the definition and the consistency of $\mathcal{M}$. This can be achieved incrementally by deforming locally a surface $S_{d}$ that bounds two regions $R_{a}$ and $R_{b}$ (Fig. 7). Preserving the model validity throughout the modification requires keeping the number and the names of the regions of $\mathcal{M}$ constant. Let $S_{f}$ denote the fixed interface defined by: $S_{f}=\left(\partial R_{a} \cup \partial R_{b}\right) \backslash S_{d}$. Then, model validity is ensured by the following deformation constraints:

Constraint 1 the borders of $S_{d}$ can only be displaced continuously along the fixed surface $S_{f}$

Constraint $2 S_{d}$ may only move inside $R_{a} \cup R_{b}$.

Constraint 2 has been extensively addressed previously by interference detection algorithms (Snyder and others, 1993, Jiménez, Thomas, and Torras, 2001). Constraint 1 can be enforced piecewise by parameterizing the fixed surface $S_{f}$ locally, and reshaping the border of $S_{d}$ in the parametric domain. The model is then updated by constrained Delaunay triangulation. The method involves a specific topological model introduced in Caumon, Sword, and Mallet (2003) and further explained in the Appendix. 


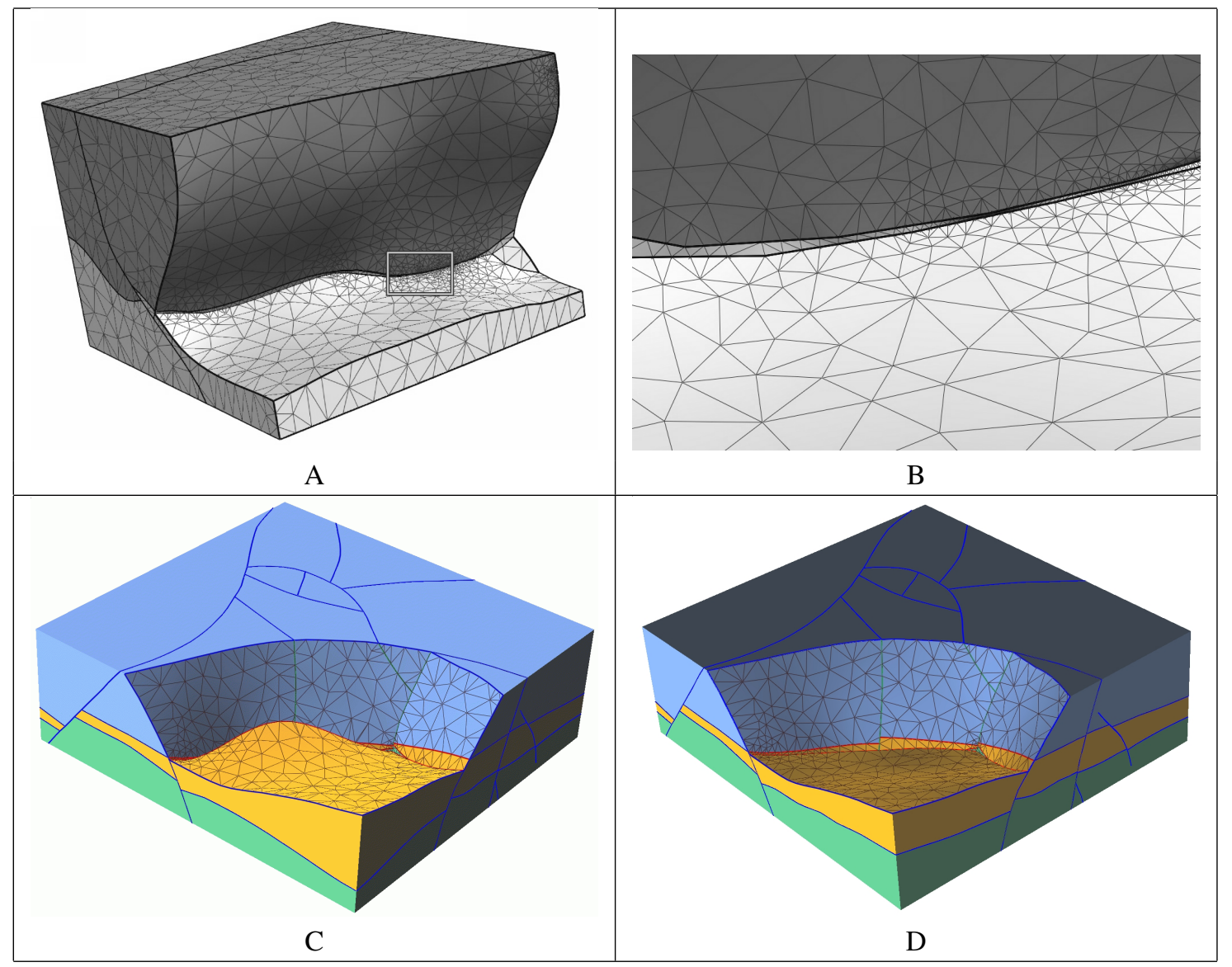

Figure 8. Two Sealed Geological Models (courtesy of Total). A and B: this model is made of 4 surfaces and 8178 triangles; note the nice mesh quality obtained by the conforming Delaunay triangulation. C and D: this model of 14 surfaces and 12500 triangles has been modified locally in real time by two simple graphic manipulations.

\section{RESULTS}

The concepts and methods presented in this article have been implemented in a C++ "plugin" on top of the Gocad modeling software. Two examples of sealed geological models obtained with this plugin are shown in Figure 8 Both models were created interactively from a set of independent surfaces in less than 15 minutes. The editing of the second model (Fig. 8-C,D) was achieved in real time on a $1 \mathrm{GHz}, 512 \mathrm{MB}$ PC.

In the first model, the artificial high density of triangles in the magnified zone (Fig. 8.B) is due to the use of a conforming Delaunay triangulation method (Lepage, 2002). Note that this could be avoided using a constrained Delaunay approach (Chew, 1989), at the expense of mesh quality. 


\section{CONCLUSION}

We have extended the notion of boundary representation to model geological structures. For that purpose, new validity conditions have been introduced and a contruction and an editing method have been defined.

In this work, we have tried to remain as general as possible, introducing only necessary validity conditions. For practical applications, however, additional validity criteria could be added according to the geological setting, as proposed for instance by Gratier and Guillier (1993); Thibault and others (1996); Ramsay and Lisle (2000).

As uncertainty is usually associated with the initial data that the models are built from; it would be interesting to couple the techniques described in this article with the stochastic simulation of geological interface geometries, as in Lecour and others (2001). Maintaining automatically the consistency of the solid model would then provide an integrated means to assess structural uncertainties (e.g., volume computations, well planning, etc.).

Most physical processes can be modeled by solving partial differential equations. Unfortunately, the numerical solvers of these equations require a discretization of space. A Sealed Geological Model provides a frame for generating such a discretization by unstructured gridding; Lepage (2002). In such a context, an interesting application of this work would be to couple gridding algorithms with this frame, for fast updates of a mesh after a model deformation (Mello and Henderson, 1997; François and Cuillière, 2000).

\section{ACKNOWLEDGEMENTS}

This work was performed in the frame of the Gocad research consortium. The authors therefore want to thank the sponsors of this consortium, and especially ChevronTexaco (which funded the Ph.D. of G. Caumon) and Shell (which supports the Ph.D. of Francois Lepage). We are indebted to Nicolas Euler (Earth Decision Sciences) and Bruno Lévy (INRIA Lorraine), without whom this work would not have been possible. Thanks to Eric De Kemp (Geological Survey of Canada) and the reviewers of this paper for their remarks and suggestions.

\section{REFERENCES}

Assa, S., Celnicker, G. and Ramshorn, C., 1998, Feature-based geometric modeling for geoscience, in Royer, J.-J., ed., 3D Modeling of Natural Objects: A Challenge for the 2000's: Gocad ENSG Conference, $10 \mathrm{p}$.

Boissonnat, J.-D. and Preparata, F., 2000, Robust plane sweep for intersecting segments: SIAM Journal on Computing, v. 29, no. 5, p. 1401-1421.

Boissonnat, J.-D. and Yvinec, M., 1998, Algorithmic Geometry: Cambridge University Press, UK, 541 p. 
Bonham-Carter, G. F., 1994, Geographic Information Systems for Geoscientists: Modelling with GIS: Pergamon Press, New York, NY, 414 p.

Caumon, G., Sword, C. H. and Mallet, J.-L., 2003, Constrained modifications of non-manifold b-rep models, in Shapiro, V. and Elber, G., eds., Proc. 8th ACM Symposium on Solid Modeling and Applications: ACM Press, New York, NY, p. 310-315.

Chew, L. P., 1989, Constrained delaunay triangulations: Algorithmica, v. 4, no. 1, p. 97-108.

Courrioux, G., Nullans, S., Guillen, A., Boissonnat, J.-D., Repusseau, P., Renaud, X., and Thibaut, M., 2001, 3d volumetric modelling of Cadomian terranes (Northern Brittany, France): an automatic method using Voronoi diagrams: Tectonophysics, v. 331, p. 181-196.

de Floriani, L. and Falcidieno, B., 1988, A hierarchical boundary model for solid object representation: ACM Transactions on Graphics, v. 7, no. 1, p. 42-60.

de Floriani, L., Morando, F. and Puppo, E., 2003, Representation of non-manifold objects through decomposition into nearly manifolds parts, in Shapiro, V. and Elber, G., eds., Proc. 8th ACM Symposium on Solid Modeling and Applications: ACM Press, New York, NY, p. 304-309.

de Kemp, E. A., 1999, Visualization of complex geological structures using 3-D Bézier construction tools: Computers and Geosciences, v. 25, no. 5, p. 581-597.

Euler, N., Sword, C. H. and Dulac, J.-C., 1998, A new tool to seal a 3d earth model: a cut with constraints, in Proc. 68th Annual Meeting: Society of Exploration Geophysicists, p. 710-713.

Euler, N., Sword, C. H. and Dulac, J.-C., 1999, Editing and rapidly updating a 3d earth model, in Proc. 69th Annual Meeting: Society of Exploration Geophysicists, p. 950-953.

Fortune, S., 1995, Polyhedral modelling with exact arithmetic, in Hoffmann, C. and Rossignac, J., eds., Proc. Third Symposium on Solid Modeling and Applications: ACM Press, New York, NY, p. 225-234.

François, V. and Cuillière, J.-C., 2000, Automatic remeshing applied to model modification: Computer Aided Design, v. 32, no. 7, p. 433-444.

Gjøystdal, H., Reinhardsen, J. E. and Åstebøl, K., 1985, Computer representation of complex three-dimensional geological structures using a new solid modeling technique: Geophysical Prospecting, v. 33, no. 8, p. 11951211.

Gratier, J. and Guillier, B., 1993, Compatibility constraints on folded and faulted strata and calculation of total displacement using computational restoration (unfold program): Journal of Structural Geology, v. 15, no. 3-5, p. 391-402.

Halbwachs, Y., Courrioux, G., Renaud, X., and Repusseau, P., 1996, Topological and geometric characterization of fault networks using 3-dimensional generalized maps: Mathematical Geology, v. 28, no. 5, p. $625-656$. 
Hale, D. and Emanuel, J., 2002, Atomic meshing of seismic images, in Proc. 72nd Annual Meeting: Society of Exploration Geophysicists, p. 2126-2129.

Higashi, M., Nakano, H., Nakamura, A., and Hosaka, M., 2001, Use of topological constraints in construction and processing of robust solid models, in Anderson, D. C., ed., Proc. Sixth ACM Symposium on Solid Modeling and Application: ACM Press, New York, NY, p. 18-29.

Hoffmann, C. M., 1989, The problems of accuracy and robustness in geometric computation: IEEE Computer, v. 22 , no. 3 , p. $31-41$.

Hubeli, A., 2002, Multiresolution techniques for non-manifolds: Ph.D. thesis, ETH Zürich, 228 p.

Jiménez, P., Thomas, F. and Torras, C., 2001, 3D collision detection: A survey: Computers and Graphics, v. 25 , no. 2, p. 269-285.

Lamboglia, K., 1994, Modélisation volumique de surfaces non-manifold: Ph.D. thesis, INPL, Nancy, France, $159 \mathrm{p}$.

Lecour, M., Cognot, R., Duvinage, I., Thore, P., and Dulac, J.-C., 2001, Modeling of stochastic faults and fault networks in a structural uncertainty study: Petroleum Geoscience, v. 7, p. S31-S42.

Lepage, F., 2002, Triangle and tetrahedral meshes for geological models, in Terra Nostra, volume 04: Proc. IAMG, Berlin, p. 105-110.

Lévy, B., 1999, Topologie Algorithmique, Combinatoire et Plongement: Ph.D. thesis, INPL, Nancy, France, $202 \mathrm{p}$.

Lévy, B. and Mallet, J.-L., 1998, Non-distorted texture mapping for sheared triangulated meshes, in Computer Graphics (Proc. Siggraph.): ACM Press, New York, NY, p. 343-352.

Lévy, B., Petitjean, S., Ray, N., and Maillot, J., 2002, Least square conformal maps for automatic texture generation: ACM Transactions on Graphics (Proc. Siggraph), v. 21, no. 3, p. 362-371.

Lienhardt, P., 1989, Subdivisions of n-dimensional spaces and n-dimensional generalized maps, in Proc. Annual ACM Symposium on Computational Geometry: ACM Press, New York, NY, p. 228-236.

Mallet, J.-L., 1992, Discrete smooth interpolation: Computer-Aided Design, v. 24, no. 4, p. 263-270.

Mallet, J.-L., 1997, Discrete modelling for natural objects: Mathematical geology, v. 29, no. 2, p. $199-219$.

Mallet, J.-L., 2002, Geomodeling: Oxford University Press, New York, NY, 624 p.

Mäntylä, M., 1988, An Introduction to Solid Modeling: Computer Science Press, Rockville, MD, 401 p.

Mello, U. T. and Henderson, M. E., 1997, Techniques for including large deformations associated with salt and fault motion in basin modeling: Marine and Petroleum Geology, v. 14, no. 5, p. 551-564.

Preparata, F. and Shamos, M., 1985, Computational Geometry : An Introduction: Springer-Verlag, New-York, NY, 398 p. 
Ramsay, J. G. and Lisle, R. J., 2000, Techniques of Modern Structural Geology : Applications of Continuum mechanics in Structural Geology, volume 3: Academic Press, London, p. 701-1061.

Sakkalis, T., Shen, G. and Patrikalakis, N. M., 2000, Representational validity of boundary representation models: Computer Aided Design, v. 32, no. 12, p. 719-726.

Sederberg, T. W. and Parry, S. R., 1986, Free-Form Deformation of solid geometric models: Computer Graphics, v. 20 , no. 4 , p. $151-160$.

Snyder, J. M., Woodbury, A. R., Fleischer, K., Currin, B., and Barr, A. H., 1993, Interval methods for multi-point collisions between time-dependent curved surfaces, in Computer Graphics (Proc. Siggraph), volume 27: ACM Press, New York, NY, p. 321-334.

Sword, C. H., 1991, Building flexible interactive, geologic models, in 61st Annual International Meeting: Society of Exploration Geophysicists, p. 1465-1467.

Thibault, M., Gratier, J.-P., Léger, M., and Morvan, J.-M., 1996, An inverse method for determining three dimensional fault with thread criterion : strike slip and thrust faults.: Journal of Structural Geology, v. 18, p. $1127-1138$.

Turner, A. K., ed., 1992, Three-Dimensional Modeling with Geoscientific Information Systems, volume 354: Kluwer Academic Publishers, Dordrecht, 443 p.

Weiler, K., 1985, Edge-Based Data Structures for Solid Modeling in Curved-Surface Environments: IEEE Computer Graphics and Applications, v. 5, no. 1, p. 21-40.

Weiler, K., 1988, The radial edge structure : a topological representation for non-manifold geometric boundary modeling, in Wozny, M., McLaughlin, H., and Encarnação, J., eds., Geometric Modeling for CAD applications: Elsevier Science Publishers, Amsterdam, p. 3-36.

\section{APPENDIX: THE CORNER MAP, A TOPOLOGICAL MODEL FOR LOCAL UPDATING}

\section{Notation}

In a sealed geological model $\mathcal{M}$, consider an editable region comprised of a deformable surface $S_{d}$ and a surface $S_{f}$ whose geometry is fixed (Fig. 7):

- $\mathcal{C}_{S_{f} \cap S_{d}}$ makes up the modifiable contact between $S_{f}$ and $S_{d}$. This contact comprises the radial edges binding the border triangles of $S_{d}$ to the border triangles of $S_{f}$. During the modification, this contact must remain on the fixed surface $S_{f}$. 

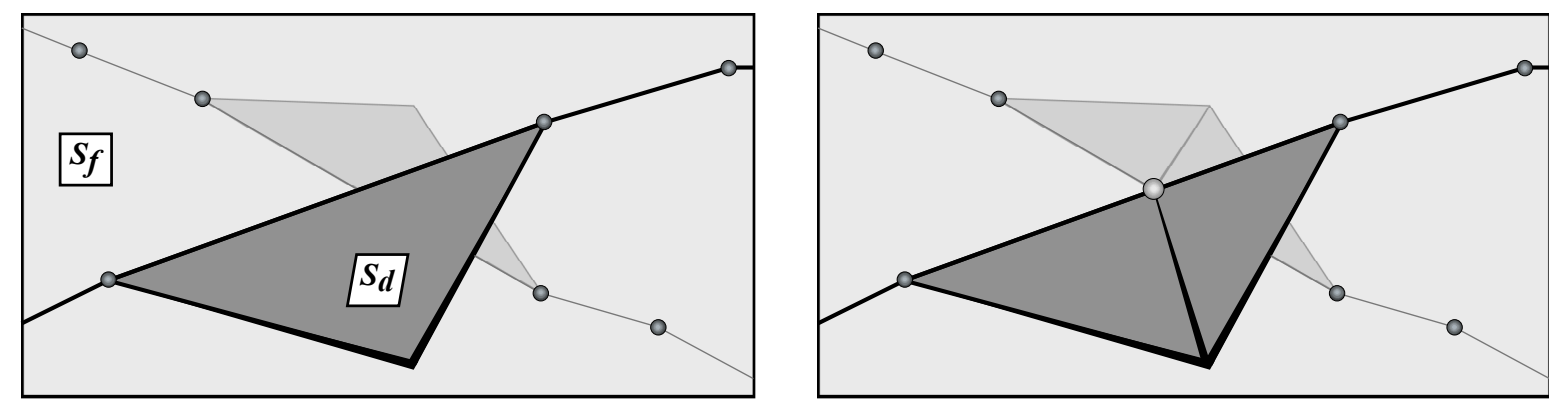

Figure 9. When contacts intersect, intersection nodes have to be inserted in or removed from both the fixed surface and the other surfaces around these contacts.
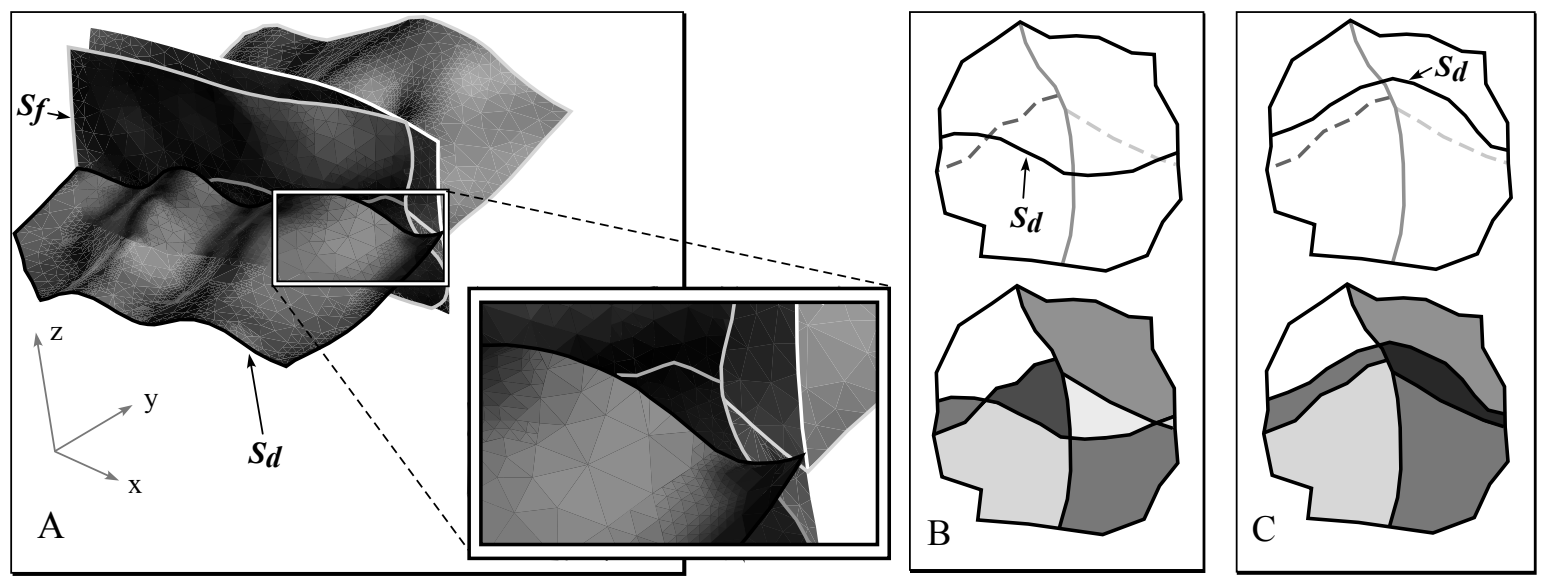

Figure 10. The deformation of $S_{d}$ may imply drastic topological changes in the model. In addition to the triangulation of interfaces, the adjacencies between polyhedral regions are subject to change.

- $\mathcal{E}_{\partial S_{f}}$ and $\mathcal{E}_{\partial S_{d}}$ consist of the other border edges of both surfaces. As these elements make up the junction of the modified domain with the remainder of the model, their geometry and topology will be fixed during the modification.

- $\mathcal{N}_{S_{d}}$ and $\mathcal{T}_{S_{d}}$ consist of the interior nodes and triangles of the interface $S_{d}$ respectively. By definition, the nodes $\mathcal{N}_{S_{d}}$ may be displaced during the modification. Their connectivities may be modified if necessary during the deformation of $S_{d}$.

- $\mathcal{N}_{S_{f}}$ and $\mathcal{T}_{S_{f}}$ consist of the interior nodes and triangles of the fixed surface $S_{f}$ respectively. These sets may be made of several manifold connected components, tied by radial edges. When the deformed interface has reached a new position, the set of triangles $\mathcal{T}_{S_{f}}$ will have to be re-computed from the original nodes $\mathcal{N}_{S_{f}}$ to account for the new contact position. 


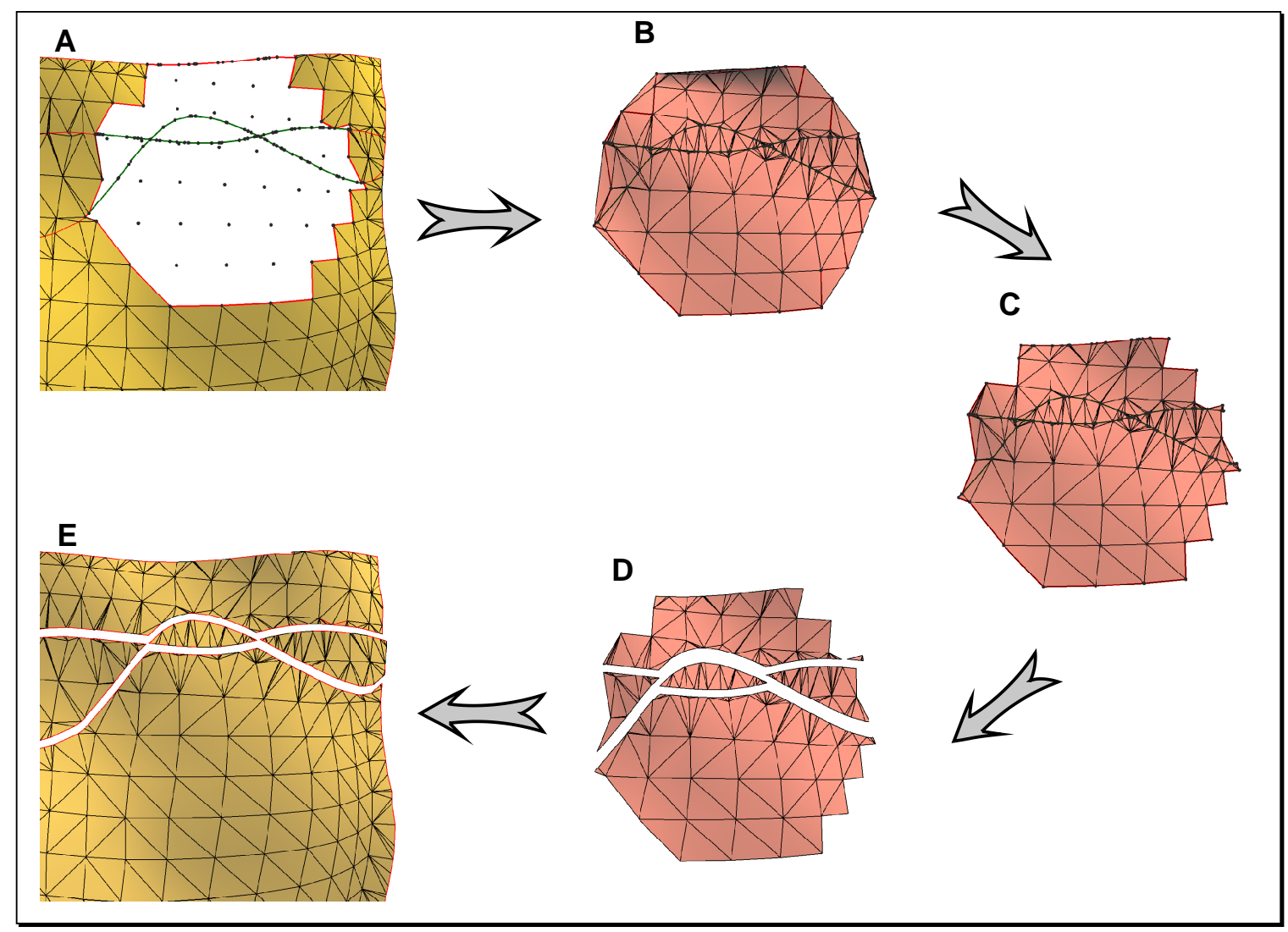

Figure 11. Updating the fixed surface $S_{f}$

\section{Accounting for topological changes}

Maintaining the model seal throughout a modification involves more than simply maintaining a geometrically sealed contact. A constrained deformation may induce significant topological changes in the model (Fig. 10). At the micro-topological level, the triangulation of the geological interfaces must be updated to honor the non-intersection condition. At the macro-topological scale, the faces of polyhedral regions may change, and region adjacencies may vary.

When this happens, intersections between the border edges of $\mathcal{E}_{\partial S_{f}}$ and of the modified contact $\mathcal{C}_{S_{f} \cap S_{d}}$ need to be inserted in or removed from the fixed surface $S_{f}$. As shown in Figure 9, the triangles around the intersecting edges must then be modified to maintain the b-rep representational validity.

The automated method to keep track of these changes proceeds as follows (Fig. 11):

1. Destroy locally the previous triangulation $\mathcal{T}_{S_{f}}$ (Fig. 11 -A).

2. Compute the wireframe intersections between $\mathcal{E}_{\partial S_{f}}$ and the modified contact $\mathcal{C}_{S_{f} \cap S_{d}}$. This can be performed using a sweep-based algorithm in parametric space (Boissonnat and Preparata, 2000).

3. Compute a Constrained Delaunay Triangulation (Chew, 1989, Lepage, 2002) of the nodes $\mathcal{N}_{S_{f}}$ in the 2D 


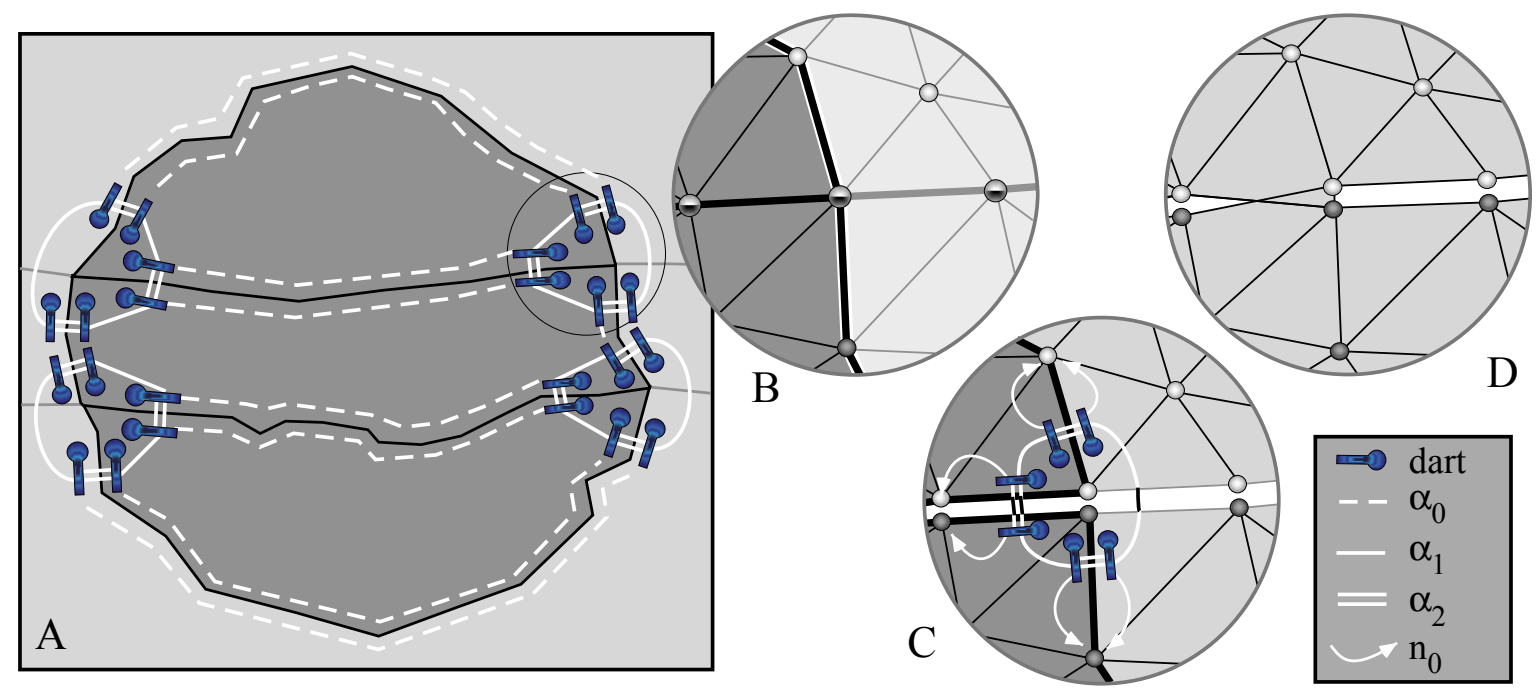

Figure 12. The corner maps of a modified patch of $S_{f}$ (A: global view; B-D: details of a corner). Gluing without caring how the new triangulation $\mathcal{T}_{S_{f}}$ is glued to the remainder of the surface may yield bad connections for co-located nodes ( $\mathrm{D}$, exploded view). A bad connection can be avoided using a corner map (A,B: normal view; C: exploded view).

parametric space. Delaunay constraints consist of all the border edges $\mathcal{E}_{\partial S_{f}} \cup \mathcal{C}_{S_{f} \cap S_{d}}$ (Fig. 11.B).

4. Clip the triangles between the boundary of the modified domain and the convex hull of $\mathcal{N}_{S_{f}}$ (Fig. 11.C).

From a non constrained border edge, these triangles are obtained by a simple graph traversal algorithm.

5. Glue the retriangulated patch to the deformed surface $S_{d}$ and to the exterior of the modified domain. (Fig. 11 .D and E).

The triangulated patch is by definition a homogeneous simplicial complex of dimension 2 without singular faces (Boissonnat and Yvinec, 1998). In other words, the patch is made of a single connected component, while the fixed surface $S_{f}$ contains co-located nodes on its internal border (Fig. 12. However, the retriangulated patch has to be unsewn along its constrained edges to meet to the non-intersection condition (Fig. 11.D).

For two co-located border nodes in the retriangulated patch, the connection to the remainder of $S_{f}$ is ambiguous (Fig. 12.D). Only topological information can be used to remove this ambiguity, but the existing model structures described so far do not provide such information. Therefore, we propose a special-purpose topological model called the corner map.

\section{The corner map}

A corner $c$ is a singular group of nodes, where three or more constrained edges meet (Fig. 12. The goal of a corner map $\operatorname{cmap}(c)$ is to identify the sectors of surface around $c$. For that, we consider the constrained edges 
on $S_{f}$ to define a polygonal complex (or 2-dimensional cellular complex), that can be conveniently represented by a 2-G-Map (Lienhardt, 1989, Halbwachs and others, 1996; Mallet, 2002) $G=\left\{\mathcal{D}, \alpha_{0} \alpha_{1}, \alpha_{2}\right\}$, where :

- $\mathcal{D}$ is a finite set of darts. In a $p$-dimensional cellular complex, a dart $d$ is defined by a series of cells of increasing dimensions $d=\left\{c_{0}, c_{1}, \ldots, c_{p}\right\}$, such that $c_{i-1} \subset \partial c_{i}, \forall i \in[1, p]$. In two dimensions $(p=2)$, we will represent geometrically a dart $d=\left\{c_{0}, c_{1}, c_{2}\right\}$ as a bullet-headed segment inside the polygon $c_{2}$, oriented along the edge $c_{1}$, and whose head is close to the vertex $c_{0}$ (Fig. 12.A-C).

- $\alpha_{0}, \alpha_{1}$ and $\alpha_{2}$ are involutions on $\mathcal{D}$ such that, for $i \in[0,2], \alpha_{i}$ links two darts $d=\left\{c_{0}, c_{1}, c_{2}\right\}$ and $d^{\prime}=\left\{c_{0}^{\prime}, c_{1}^{\prime}, c_{2}^{\prime}\right\}$ differing only by one cell of dimension $i$

$$
\alpha_{i}(d)=d^{\prime} \quad \Longleftrightarrow \quad \begin{aligned}
& c_{i \oplus 1}=c_{i \oplus 1}^{\prime} \\
& c_{i \oplus 2}=c_{i \oplus 2}^{\prime}
\end{aligned}
$$

where $i \oplus j=(i+j) \% i$ is the residual of the integer division of $i+j / i$

From this definition, the corner map $\operatorname{cmap}(c)=\left\{G, n, v_{0}\right\}$ is obtained as a specialization of the 2-G-Map $G$, where $n$ and $v_{0}$ are functions that associate a dart of $\mathcal{D}$ to a surface node of $\mathcal{N}_{S_{f}}$. For any pair of darts $\left(d_{1}, d_{2}\right) \in \mathcal{D}^{2}:$

$$
\begin{aligned}
\alpha_{1}\left(d_{1}\right)=d_{2} & \Longleftrightarrow n\left(d_{1}\right)=n\left(d_{2}\right) \\
\alpha_{2}\left(d_{1}\right)=d_{2} & \Longleftrightarrow\left\{\begin{array}{l}
n\left(d_{1}\right) \neq n\left(d_{2}\right) \\
n\left(d_{1}\right) \text { and } n\left(d_{2}\right) \text { are co-located } \\
v_{0}\left(d_{1}\right) \text { and } v_{0}\left(d_{2}\right) \text { are co-located }
\end{array}\right.
\end{aligned}
$$

The corner map can be built from the standard model data structures before editing. After the triangulation and the unsewing operations, the inverse function $n^{-1}$ is used to access the corner map whenever corner nodes are detected.

With this topological model, the new topology of the fixed surface $S_{f}$ can therefore be correctly computed, and the radial contact information successfully updated. As the number of regions is invariant through the modification, the layer definitions and the associated information can be preserved. 